\title{
Dilaton-spin dark matter in Cartan-Weyl space
}

\section{Babourova O. V., Frolov B.N., Zaigrova V.V.}

Moscow State Pedagogical University, Institute of Physics, Technology and Information Systems, Moscow, Russian Federation;

E-mail: Babourova <baburova@orc.ru>,Frolov<frolovbn@orc.ru>,Zaigrova <zaigrova07@list.ru>;

The perfect dilaton-spin fluid, as a model of the dilaton matter, the particles of which are endowed with intrinsic spin and dilaton charge, is considered as the source of the gravitational field in a Cartan-Weyl spacetime. The variational equations of the theory are deduced in a formalism of external forms. The solution of these equations is obtained for the homogeneous and isotropic ultra early universe.

Keywords: Dilaton-spin fluid, variational principle, Cartan-Weyl space.

DOI: $10.18698 / 2309-7604-2015-1-60-66$

\section{Dilaton-spin dark matter}

The modern observations in cosmology lead to the following conclusions:

The first one is about existence of dark matter with the density exceeding by one order of magnitude the density of baryonic matter.

The second conclusion consists in preposition that dark matter interacts with the equal by order of magnitude positive vacuum energy (or quintessence).

And the third conclusion is the understanding of the fact that the expansion with deceleration is succeeded by the expansion with acceleration.

In [1] it was constructed a model of a dark matter as a dilaton-spin fluid representing an ideal fluid with the additional degrees of freedom. Each particle of such fluid is endowed with the dilaton-spin tensor $J_{q}^{p}$,

$$
J_{q}^{p}=S_{q}^{p}+\frac{1}{4} \delta_{q}^{p} J, S_{q}^{p}=J_{q]}^{[p}, J=J_{p}^{p}
$$

It's antisymmetric part $S_{q}^{p}$ is the spin tensor. The second term is proportional to the specific (per particle) dilaton charge $J$ of the fluid element. $J$ is the trace of the dilaton-spin tensor.

As a result of the variational procedure we get the canonical energy-momentum 3-form

$$
\sum_{a}=p \eta_{a}+(\varepsilon+p) u_{a} u+n \dot{S}_{a b} u^{b} u
$$


the metric energy-momentum 4-form

$$
\sigma^{a b}=T^{a b} \eta, T^{a b}=p g^{a b}+(\varepsilon+p) u^{a} u^{b}+n \dot{S}_{c}^{(a} u^{b)} u^{c}
$$

and the dilaton-spin tensor $J_{q}^{p}(1.1)$. In (1.2), (1.3) $\varepsilon$ is the internal energy density of the fluid, $p$ is the hydrodynamic fluid pressure, $n$ is the fluid particles concentration equal to the number of fluid particles per a volume unit, $\eta$ is a volume 4-form, $\eta_{a}$ is a 3 -form defined as $\left.\left.\eta_{a}=\bar{e}\right\rfloor \eta=* \theta_{a}.\right\rfloor$ means the interior product, $*$ is the Hodge dual operator, $\theta^{a}$ is a cobasis of 1-forms of the Cartan-Weyl space. Each fluid element possesses a 4-velocity vector $\bar{u}=u^{a} \bar{e}_{a}$ which is corresponded to a flow 3 -form $\mathrm{u}, u=\bar{u}\rfloor \eta=u^{a} \eta_{a}$ and a velocity 1 -form $* u=u_{a} \theta^{a}$. In (3) the "dot" notation for the tensor object $\Phi^{a}{ }_{b}$ is introduced, $\dot{\Phi}_{b}^{a}=*\left(u \wedge D \Phi_{b}^{a}\right), \quad \mathrm{D}=\mathrm{d}+\Gamma \wedge \ldots$ is the exterior covariant differential, $\wedge .-$ the exterior product operator.

\section{Cartan-Weyl space}

The basic concept of the modern fundamental physics consists in proposition that spacetime geometrical structure is compatible with the properties of matter filling the spacetime. As a result of this fact the matter dynamics exhibits the constraints on a metric and a connection of the spacetime manifold. Dilaton-spin matter generates in spacetime the Cartan-Weyl $C W_{4}$ geometrical structure with curvature $\mathcal{R}^{a}{ }_{b}$, torsion $\mathcal{T}^{a}$ and nonmetricity $\mathcal{Q}_{a b}$ of the Weyl type.

Let us consider a connected 4-dimensional oriented differentiable manifold $\mathcal{M}$ equipped with a metric $g_{a b}$ of index 1 , a connection 1 -form $\Gamma_{b}^{a}$ and a volume 4-form $\eta$. Then Cartan-Weyl $C W_{4}$ space is defined as such manifold equipped with a curvature 2-form

$$
\mathcal{R}_{b}^{a}=d \Gamma_{b}^{a}+\Gamma_{c}^{a} \wedge \Gamma_{b}^{c}, \mathcal{R}_{b}^{a}=\frac{1}{2} \mathcal{R}_{b c d}^{a} \theta^{c} \wedge \theta^{d}
$$

and torsion 2-form 


$$
\mathcal{T}^{a}=d \theta^{a}+\Gamma_{b}^{a} \wedge \theta^{b}, \mathcal{T}^{a}=\frac{1}{2} T_{c d}^{a} \theta^{c} \wedge \theta^{d}
$$

The metric tensor and the connection 1-form obey the Weyl condition,

$$
\begin{aligned}
& \mathcal{Q}=g^{a b} \mathcal{Q}_{a b}, \mathcal{Q}=\mathcal{Q}_{a} \theta^{a}, \quad \mathcal{Q}_{a b}=Q_{a b \mu} h_{c}^{\mu} \theta^{c}, \\
& Q_{a b \mu}=-\nabla_{\mu} g_{a b}, \mathcal{Q}_{a b}=-D g_{a b}=(1 / 4) g_{a b} \mathcal{Q},
\end{aligned}
$$

where $\mathcal{Q}_{a b}$ is a nonmetricity 1 -form, $\mathcal{Q}$ is a Weyl 1 -form.

\section{Variational formalism in Cartan-Weyl space}

In [2] the modified variational formalism is advanced, according to which the Weyl-Dirac scalar field $\beta$ is introduced in the Lagrangian density, but not entered by "hands" irrespective of the metric (how it was carried out in [3], [4]), but is entered as a representation of a tangent space metric as

$$
g_{a b}=\beta^{2} g_{a b}^{M}, \mathcal{Q}=q d \ln \beta, q=-8
$$

where $g_{a b}^{M}$ are constant components of Minkowski metric and an arbitrary function of spacetime points $\beta(x)$ describes some scalar field of geometrical nature. Such a metric tensor representation follows from the Poincare-Weyl gauge theory, advanced in [5], and corresponds to a lemma (to B.N. Frolov, 2003) about a metric tensor of a general affine-metric space, proved in [6].

The representation (3.1) enters in the Lagrangian density with the help of a method of uncertain Lagrange multipliers. We represent the total Lagrangian density 4-form of the theory as the sum of the Lagrangian density 4-form of the gravitational field $\mathcal{L}_{G}$ and of the dilaton-spin fluid $\mathcal{L}_{f}: \mathcal{L}=\mathcal{L}_{G}+\mathcal{L}_{f}$, while the Lagrangian density 4-form of the gravitational field reading. 


$$
\begin{aligned}
& \mathcal{L}_{G}=2 f_{0}\left((1 / 2) \mathcal{R}^{a}{ }_{b} \wedge \eta_{a}{ }^{b}+\rho_{1} \mathcal{T}^{a} \wedge * \mathcal{T}_{a}+\right. \\
& +\rho_{2}\left(+\mathcal{T}^{a}++\wedge \theta_{b}\right) \wedge *\left(\mathcal{T}^{b} \wedge \theta_{\alpha}\right)+\rho_{3}\left(\mathcal{T}^{a} \wedge \theta_{a}\right) \wedge *\left(\mathcal{T}^{b} \wedge \theta_{b}\right)+ \\
& \left.+\xi \mathcal{Q} \wedge * \mathcal{Q}+\zeta \mathcal{Q} \wedge \theta^{a} \wedge * \mathcal{T}_{a}\right)-\Lambda \eta+ \\
& +\Lambda^{a b} \wedge\left(\mathcal{Q}_{a b}-(1 / 4) g_{a b} \mathcal{Q}\right)+\lambda^{a b}\left(g_{a b}-\beta^{2} g_{a b}^{M}\right) \eta
\end{aligned}
$$

Here $\eta_{a}{ }^{b}=*\left(\theta_{a} \wedge \theta^{b}\right), \mathrm{f}_{0}=1 /(2 æ) \quad(æ=8 \pi G)$. The first term is the linear Hilbert-Einstein Lagrangian generalized to the Cartan-Weyl space. $\Lambda$ is the cosmological constant, $\lambda, \rho_{1}, \rho_{2}$, $\rho_{3}, \xi, \zeta$ are the coupling constants, $\Lambda^{a b}$ and $\lambda^{a b}$ are the Lagrange multipliers, and we have as consequence of the Weyl condition (2.3), $g_{a b} \Lambda^{a b}=0$.

We use the variational procedure in the exterior form language which is based on the master formula derived the following Lemma, proved in [7]. Lemma gives the rule how to compute the commutator of the variation operator $\delta$ and the Hodge star operator.

The variation of the total Lagrangian density 4-form with respect to the connection 1-form $\Gamma_{b}^{a}$ and to the base 1 -form $\theta^{a}$ gives $\Gamma$-equation and $\theta^{a}$-equation. The variation to the components of the tangent space metric $g_{a b}$ gives $g$-equation. The variation of the Lagrangian density 4-form with respect to the Lagrange multipliers $\Lambda^{\alpha \beta}$ and $\lambda^{a b}$ yields the Weyl condition (2.3) and the representation (3.1). The variation to the scalar field $\beta$ gives the relation, $\lambda^{a b} g_{a b}=0$

After antisymmetrization of the $\Gamma$-equation and then contracting externally on the right with $\theta^{a}$, we receive the consequence of this equation, which is not containing the Lagrange uncertain multipliers,

$$
\frac{2}{3}\left(1-\rho_{1}+2 \rho_{2}\right) \mathcal{T}=\left(\frac{1}{4}+\zeta\right) \mathcal{Q}
$$

where $\mathcal{T}=*\left(\theta_{c} \wedge * \mathcal{T}^{c}\right)$ is a torsion trace 1 -form. After contracting the $\Gamma$-equation and using (3.3), one obtains another consequence, 


$$
Q^{a}=\frac{æ}{2 \tau} n J u^{a}, \tau=16 \xi+\frac{3\left(\rho_{1}-2 \rho_{2}+8 \zeta(1+2 \zeta)\right)}{4\left(1-\rho_{1}+2 \rho_{2}\right)} .
$$

\section{Modified Friedmann-Lemaitre equation}

It was proved that in the homogeneous and isotropic universe with Friedmann-Robertson-Walker (FRW) metric

$$
d s^{2}=\frac{a^{2}(t)}{1-k r^{2}} d r^{2}+a^{2}(t) r^{2}\left(d \theta^{2}+(\sin \theta)^{2} d \varphi^{2}\right)-d t^{2}
$$

filled with the dilaton dark matter (for FRW metric $S_{a b}=0$ ) and dark energy described by the cosmological term $\Lambda$, the Einstein-like equation is valid:

$$
\begin{gathered}
\left(\begin{array}{c}
R^{a} \\
R_{b}-\frac{1}{2} \delta_{b}^{a} R
\end{array}\right) \eta_{a}+\Lambda \eta_{b}+\alpha\left(2 Q_{b} Q^{a} \eta_{a}-Q_{a} Q^{a} \eta_{b}\right)=æ \Sigma_{b}, \\
\alpha=\frac{3\left(\frac{1}{4}+\zeta\right)^{2}}{4\left(1-\rho_{1}+2 \rho_{2}\right)}+\xi-\frac{3}{64} .
\end{gathered}
$$

Here $\stackrel{R}{R}_{\sigma}^{\rho}$ and ${ }^{R}$ are a Ricci tensor and a scalar curvature of a Riemannian space respectively. As a consequence of (2.3) and (3.1) the thirt term in (4.2) using (3.4) can be rewritten in the form of an energy-momentun tensor of a perfect fluid,

$$
T_{a b}=\left(\varepsilon_{\beta}+p_{\beta}\right) u_{a} u_{d}-p_{\beta} g_{a b}, \varepsilon_{\beta}=p_{\beta}=\alpha æ\left(\frac{n J}{2 \tau}\right)^{2}
$$

After integrating the continuity equation $\mathrm{d}(n u)=0$ for the FRW metric one obtains the matter conservation law $n a^{3}=N=$ const .

Therefore, taking into account (3.4), the equation (4.2) can be represented in the form, 


$$
R_{a b}-\frac{1}{2} g_{a b} R=æ\left(\left(\varepsilon_{\mathrm{e}}+p_{\mathrm{e}}\right) u_{a} u_{b}-p_{\mathrm{e}} g_{a b}\right)
$$

where $\varepsilon_{\mathrm{e}}$ and $p_{\mathrm{e}}$ are an energy density and a pressure of an effective perfect fluid,

$$
\varepsilon_{\mathrm{e}}=\varepsilon+\varepsilon_{\mathrm{v}}-E\left(\frac{n}{N}\right)^{2}, \quad p_{\mathrm{e}}=p+p_{\mathrm{v}}-E\left(\frac{n}{N}\right)^{2}, E=\alpha æ\left(\frac{J N}{2 \tau}\right)^{2}
$$

Here $\varepsilon_{\mathrm{v}}=\Lambda / \mathfrak{x}$ and $p_{\mathrm{v}}=-\Lambda / \mathfrak{x}$ are an energy density and a pressure of a vacuum with the equation of state, $\varepsilon_{\mathrm{v}}=-p_{\mathrm{v}}>0$.

The equation (4.3) yields the modified Friedman-Lemaitre (FL) equation

$$
\left(\frac{\dot{a}}{a}\right)^{2}+\frac{k}{a^{2}}=\frac{æ}{3}\left(\varepsilon+\varepsilon_{\mathrm{v}}-\alpha æ\left(\frac{J n}{2 \lambda m^{2}}\right)^{2}\right) .
$$

Let us consider the case of super early Universe, when the scalar field $\beta$ is very intensive and according to (4.4) has an equation of state of super-rigid matter. If we suppose that in this case the dilaton-spin fluid also has its equation of state of super-rigid matter, then the modified Friedman-Lemaitre equation has the form

$$
\left(\frac{\dot{a}}{a}\right)^{2}=\frac{æ}{3 a^{6}}\left(\varepsilon_{v} a^{6}+\mathcal{E}_{1}-\mathcal{E}\right), \quad k=0,
$$

where $\mathcal{E}_{1}=\varepsilon a^{6}=$ const is the integration constant of the energy conservational law

$$
d \varepsilon=\frac{\varepsilon+p}{n} d n
$$


The equation (4.6) can be exactly integrated after representing in the form

$$
\left(\frac{\dot{a}}{a}\right)^{2}=\frac{\Lambda}{3 a^{6}}\left(a^{6}-a_{\min }^{6}\right), \quad a_{\min }=\left(\frac{\alpha æ^{2}}{\Lambda}\left(\frac{J N}{2 \tau}\right)^{2}-\frac{\mathcal{E}_{1} æ}{\Lambda}\right)^{1 / 6}
$$

The solution corresponding to the initial data $t=0, a=a_{\min }$ reads,

$$
a=a_{\min }(\cosh \sqrt{3 \Lambda} t)^{1 / 3}
$$

This solution describes the inflation-like stage of the evolution of the Universe, which continues while the scalar field $\beta$ will reduce its intensity, and the equation of state of the dilaton matter will change and will become differ from the equation of state of the super-rigid matter.

The results were obtained within the framework of performance of the state task No 3.1968.2014/K of the Ministry of education and science of the Russian Federation.

\section{References}

1. Babourova O. V., Frolov B. N. (2003). Class. Quantum Grav., V. 20, 1423-1441.

2. Babourova O. V., Frolov B. N., Febres E. V. (2015). Russ. Phys. Journal, V. 58, N 2, $283-285$.

3. Babourova O. V., Frolov B. N. (2012). Mathematical foundations of the modern theory of gravitation, MPGU, Moscow.

4. Babourova O. V., Frolov B. N., Lipkin K. N. (2012). Gravit. Cosm., V. 18, No 4, 225-231.

5. Babourova O. V., Frolov B. N., Zhukovsky V. Ch. (2006). Phys. Rev. D., V. 74, 064012-1-12.

6. Frolov B.N. (2003). Poincare gauge gravitational theory. Moscow: MPGU.

7. Babourova O. V., Frolov B. N., Klimova T. A. (1999). Class. Quantum Grav., V. 16, 1-14. 